\title{
Wilhelm Weber und Maxwells elektromagnetische Lichttheorie $^{1}$
}

\author{
Karl Heinrich Wiederkehr
}

\section{Summary}

This essay aims at demonstrating a possibly decisive impulse which induced J. Cl. Maxwell to create his electromagnetical theory of light. With their experiment in $1855 R$. Kohlrausch and $W$. Weber had come across the velocity of light when determining the relation between the absolute electrostatic and the absolute electromagnetic quantity of electricity which they had measured. In Maxwell's treatise "On Physical Lines of Force" (1861/62) that relation is exactly identical with the velocity of transmission of possible transversal waves in Maxwell's hypothetical medium (ether). The experiment of 1855, a masterpiece, is described in detail. The " $v$-determinations" which were later carried out by some physicists - among them Maxwell himself and W.Thomson - verified the convergency with the more accurate measurements of the velocity of light. Thus the validity of the electromagnetical theory of light was secured.

\section{ZuSAMMENFASSUNG}

Diese Abhandlung will an einen vielleicht entscheidenden Anstoss erinnern, der J.Cl. Maxwell zur Schaffung seiner elektromagnetischen Lichttheorie bewogen hat. R. Kohlrausch und W. Weber waren bei ihrem Experiment von 1855 auf die Lichtgeschwindigkeit gestossen, als sie das Verhältnis von absolut elektrostatisch und absolut elektromagnetisch gemessener Elektrizitätsmenge bestimmten. In der Maxwellschen Arbeit "Über Physikalische Kraftlinien» (1861/62) ist gerade jenes Verhältnis identisch mit der Fortpflanzungsgeschwindigkeit möglicher Transversalwellen in Maxwells hypothetischem Medium (थ̈ther). Das Experiment von 1855 stellt eine Meisterleistung dar und wird genauer geschildert. Die später von mehreren Physikern - darunter Maxwell selbst und $W$. Thomson - durchgeführten " $v$-Bestimmungen» bestätigten die Konvergenz mit den genaueren Lichtgeschwindigkeitsmessungen. Die Gültigkeit der elektromagnetischen Lichttheorie war damit gesichert. 
Nach Beendigung seines 2. Teiles der Abhandlung «Physical Lines of Force» (1861) beschäftigte sich James Clerk Maxwell (1831-1879) weiterhin intensiv mit den Eigenschaften des von ihm gebrauchten Mediums, des Äthers. Dieses Medium wurde von ihm paradoxerweise wie ein fester, hochelastischer Körper behandelt, in dem auch transversale Wellen möglich sind. Für ihre Fortpflanzungsgeschwindigkeit fand Maxwell die Formel $\mathbf{v}=\sqrt{\frac{m}{\varrho}}$, worin $\mathrm{m}$ das Schermodul und $\varrho$ die Dichte ist. ${ }^{2}$ Seine Betrachtungen über die wirksamen Kräfte beim Coulombschen Gesetz der Elektrostatik führten ihn zum Verhältnis absolut elektrostatisch und absolut elektromagnetisch gemessener Elektrizitätsmengen, und nach seinen Berechnungen ist dieses Verhältnis, das die Dimension einer Geschwindigkeit besitzt, identisch mit der Fortpflanzungsgeschwindigkeit der Transversalwellen. Rudolf Kohlrausch (1809-1858) und Wilhelm Weber (1804-1891) hatten diese Grösse bereits 1855 in einem Experiment bestimmt. ${ }^{3}$ Sie entsprach fast der von Fizeau 1849 zum erstenmal terrestrisch gemessenen Lichtgeschwindigkeit. ${ }^{4}$ In höchster innerer Erregung schrieb Maxwell am 19. Oktober 1861 an M. Faraday: ${ }^{5}$

\footnotetext{
"From the determination by Kohlrausch and Weber of the numerical relation between the statical and magnetic effects of electricity, I have determined the elasticity of the medium in air, and assuming that it is the same with the luminiferous ether, I have determined the velocity of propagation of transverse vibrations.

The result is 193,088 miles per second (deduced from electrical and magnetic experiments). Fizeau has determined the velocity of light $=193,118$ miles per second, by direct experiment.

This coincidence is not merely numerical.... I think we have now strong reason to believe, whether my theory is a fact or not, that the luminiferous and electromagnetic medium are one."
}

Welch wichtige Rolle das Kohlrausch-Weber-Experiment bei Maxwell am Beginn seiner elektromagnetischen Lichttheorie spielte, wird bei Physikern und Physikhistorikern meist übersehen. So sucht man z. B. in den heute oft benutzten «Lectures on Physics» von R. P. Feynman und in der 1981 erschienenen Maxwell-Biographie von I. Tolstoy vergeblich nach diesem so wichtigen Anstoss. ${ }^{6}$

Wie kam es dazu, dass zwei so wichtige unterschiedliche Wissenszweige wie Optik und Elektrizitätslehre in ein- und derselben Theorie vereinigt werden konnten? Zur Beantwortung möge etwas weiter ausgeholt werden. Beeinflusst von dem Gedanken der romantischen Naturphilosophie, dass alle Naturkräfte wie Magnetismus, Elektrizität, Wärme und Licht sich 
beinflussen und wechselseitig sich umwandeln können, suchte Faraday schon früh nach der Verwandlung von Elektrizität in Licht. Ende 1845 fand er mit dem Stück Bleiglas, an dem er den Diamagnetismus entdeckt hatte, endlich einen solchen Effekt: Ein magnetisches Kraftfeld konnte die Polarisationsebene des Lichtes, das durch das Bleiglas hindurch ging, drehen. Faradays Entdeckung wurde als Sensation empfunden und zog zahlreiche Untersuchungen anderer Forscher nach sich. Im dritten Jahrzehnt des vergangenen Jahrhunderts hatte sich mit Thomas Young, Augustin Jean Fresnel und François Arago endgültig die Wellentheorie des Lichtes gegen die Korpuskular- oder Emissionstheorie durchgesetzt. Und um die vielfältigen Erscheinungen, die nach der Entdeckung der Polarisation des Lichts durch Malus 1808 gefunden worden waren, erklären zu können, war man gezwungen, den Lichtwellen transversalen Charakter zuzuerkennen. Für die «Emissionisten» wie Laplace und Biot blieb dies eine Absurdität. Wie sollte auch dem lichttragenden Medium, dem Äther, Eigenschaften zugeschrieben werden können, die man sonst nur bei hochelastischen festen Körpern, wie z.B. Stahl, antrifft, und die Weltkörper sich in diesem Medium doch ohne jeglichen Widerstand bewegen können? 1856 hatte W.Thomson (18241907), der spätere Lord Kelvin, versucht, Faradays magneto-optischen Effekt mit Hilfe torsionselastischer Eigenschaften des Äthers zu erklären. Er zog dazu eine ältere Idee von W. J.M. Rankine (1820-1872) heran, nämlich die der Molekularwirbel (Molecular vortices), eine Art von Atmosphären, die um einen Kern rotieren. Rankine wollte damit thermodynamische Eigenschaften der Gase deuten. Maxwell nahm nun in der oben erwähnten Abhandlung (1861/1862) diese Idee der Molekularwirbel auf, um die Eigenschaften des magnetischen Kraftfeldes von Faraday zu verdeutlichen. Er hatte sich vorgenommen, den Faradayschen Feldvorstellungen, die von den Zeitgenossen meist nicht ernst genommen wurden, mathematische Gestalt zu geben unter Benutzung mechanischer Analogien. Die magnetischen und elektrischen Kraftlinien stellten nach Faraday eine kontinuierliche Aneinanderreihung infinitesimal kleiner magnetischer und elektrischer Dipole dar. Längs einer Feldlinie herrscht Zug, quer zu den Feldlinien ein Druck. Maxwell konnte nun mit einer Zellenstruktur des Äthers diese Kraftwirkungen verständlich machen. Der Inhalt der Zellen ist eine elastische Substanz, durch ihre Rotation werden die magnetischen Kräfte hervorgebracht. Diese Zellen waren zunächst sechseckige Säulen, deren Achsen in Feldlinienrichtung liegen. Später gab Maxwell diesen Zellen Kugelgestalt. Der Drehsinn bestimmt die Richtung der Polarisation und damit die Lage 
von Nord- und Südpol. Die Zentrifugalkräfte verursachen die Querkraft der Feldlinien. Die Sache hat aber einen Haken: Die aneinander vorbeilaufenden Flächen der Molekularwirbel haben nämlich entgegengesetzte Bewegungsrichtungen. Maxwell bringt deswegen in die Zwischenräume kleine kugelförmige Teilchen, die die Funktion von Friktionsrollen oder Differential- und Planetenrädern haben. Und diese Friktionsrollen - das ist der Clou dieser Idee - sind zugleich die elektrischen Teilchen, die überall vorhanden sind, im Äther, in Isolatoren und Leitern. So wurden gewissermassen zwei Fliegen mit einer Klappe geschlagen. In Nichtleitern sind diese Teilchen nur verschiebbar; hier liegt die so wichtige Grundidee des Verschiebungsstromes vor. In Leitern dagegen können sich diese elektrischen Teilchen gegen einen Widerstand fortbewegen. Maxwell kann mit dieser komplizierten Äthermaschinerie, die aus einer Art ineinandergreifenden Zahnrädern und Zahnstangen besteht, alle magnetischen und elektrischen Erscheinungen vorerst verständlich machen. In jenen Jahren gebrauchte Maxwell noch solche mechanische Modelle; er wollte damit Vorgänge, die in ihrem gesetzlichen Ablauf «analog» waren, verständlich machen oder «illustrieren». Das Geschehen im Mikrokosmos auf diese mechanische Weise darzustellen, war typisch für die meisten englischen Physiker dieser Epoche. Derartige Modelle wirkten auf die Festlandphysiker der französischen Schule - und dazu gehörte auch W. Weber-mit ihrer Vorliebe für das Mathematisch-Abstrakte nicht überzeugend, vielleicht sogar befremdend. In jener mechanischen Denkweise sehen die Historiker einen Zusammenhang mit der aufkommenden Industrialisierung in England. Maxwell löste sich ein paar Jahre später von solchen mechanischen Vorstellungen; die gewonnenen Beziehungen und Ergebnisse behielt er aber bei. In der Abhandlung von 1861/62 sind seine berühmt gewordenen Gleichungen der Elektrodynamik im Keime schon vorhanden.

Im dritten Teil der Arbeit «Über physikalische Kraftlinien» wendet Maxwell nun seine Theorie der Molekularwirbel auf die elektrostatischen Erscheinungen an. Bei Anlegen der Spannung werden die elektrischen Teilchen (Friktionsrollen) verschoben. Die Grösse ihrer Verschiebung - sie wird in Ladung pro Fläche gemessen - ist aber nicht nur von der elektrischen Feldstärke abhängig, sondern auch von der Beschaffenheit des Dielektrikums, genauer gesagt, von dessen Permittivität (Dielektrizitätskonstante). Die Dielektrizitätskonstante ist so mit den elastischen Konstanten des Mediums verknüpft. Schliesslich gelangt Maxwell in seinen Überlegungen zu dem Coulombschen Gesetz der Elektrostatik. In ihm sind zunächst die 
Elektrizitätsmengen im elektromagnetischen Masse enthalten. Wird nun die Elektrizitätsmenge im Coulomb-Gesetz in elektrostatischem Masse ausgedrückt, ist nach der Definition von Gauss und Weber bekanntlich der Proportionalitätsfaktor gleich 1 zu setzen. Der Vergleich führt zum Verhältnis von elektrostatisch und elektromagnetisch gemessener Elektrizitätsmenge. Dieses Verhältnis ist aber, wie zu Anfang bereits gesagt, identisch mit der Fortpflanzungsgeschwindigkeit der transversalen elektromagnetischen Wellen im Äther.

Betrachten wir noch einmal genauer die Grössen und Dimensionen der Elektrizitätsmengen in den beiden absoluten Masssystemen. Zur absoluten elektrostatischen Ladungseinheit gelangt man über das Coulomb-Gesetz, ihre Dimension ist $\mathrm{M}^{2} \mathrm{~L}^{2} \mathrm{Z}^{-1}$ (M: Masse, L: Länge, Z: Zeit); die absoluten Systeme sind ja Dreiersysteme. Die elektromagnetisch gemessene Ladungseinheit leitet sich vom Coulomb-Gesetz der Magnetostatik (Polstärke, magnetischer Fluss) und der Äquivalenz von Kreisstrom und Stabmagnet her. Für ihre Dimension ergibt sich $\mathbf{M}^{2} \mathrm{~L}^{2} .^{7}$ Der Quotient der beiden Dimensionen hat die Dimension einer Geschwindigkeit. Nimmt man nun noch die Zahlenwerte hinzu, die sich aus dem Kohlrausch-Weber-Experiment ergeben, bekommt man $3,107 \cdot 10^{8} \mathrm{~ms}^{-1}$. Fizeau hatte für die Lichtgeschwindigkeit 3,15 $\cdot 10^{8} \mathrm{~ms}^{-1}$ gefunden.

Es ist das Verdienst Maxwells, hier sofort den Zusammenhang mit der Lichtgeschwindigkeit erkannt zu haben, und in der Abhandlung von 1861/ 62 kündigt sich seine elektromagnetische Lichttheorie an. ${ }^{8}$ Sie erschien dann zwei Jahre später und ist in der revolutionierenden Arbeit «A Dynamical Theory of the Electromagnetic Field» (1864/65) enthalten. Hierin entwickelte Maxwell nun seine berühmten Gleichungen ohne Gebrauch mechanischer Modelle und gibt, wie auch später in seinem «Treatise on Electricity and Magnetism» (1873) für die Feldgrössen der elektrischen Verschiebung, der magnetischen Induktion und des Vektorpotentials die Wellendifferentialgleichungen an. ${ }^{9}$

Wie führten R. Kohlrausch und W. Weber 1855 ihr schwieriges Experiment durch und was war der Anlass gewesen? Kohlrausch, damals ausserordentlicher Professor in Marburg, hatte in einem Vortrag den Plan dargelegt, das Webersche Gesetz einer grundsätzlichen experimentellen Prüfung zu unterziehen. Weber hatte es in seiner ersten Abhandlung der Reihe «Elektrodynamische Maassbestimmungen» aufgestellt. Der Schule der französischen Physiker folgend - sie war im ersten Drittel des 19. Jahrhunderts 
führend - war es ein sogenanntes Fernwirkungsgesetz. Die beiden Elektrizitätsarten wurden als imponderable Substanzen betrachtet. Die Elektrizitätsteilchen - atomistischen Vorstellungen folgend - wirkten ohne irgendein Zwischenmedium aus der Distanz aufeinander, ähnlich wie im Gravitationsgesetz zwei Weltkörper, und dies ohne irgendeine Verzögerung. ${ }^{10} \mathrm{Im}$ Weberschen Gesetz werden die elektrodynamischen, sprich magnetischen Kräfte, durch Glieder, die dem Coulombschen Gesetz der Elektrostatik hinzugefügt sind, erfasst, und zwar mit Hilfe der ersten und zweiten Ableitung des Abstandes $\mathrm{r}$ der beiden Teilchen nach der Zeit. Dass in einem elektrischen Kraftgesetz die Geschwindigkeit und Beschleunigung auftaucht, war neu. Eine solche Abhängigkeit tritt erst viel später in dem Kraftgesetz von H. A. Lorentz und in der Relativitätstheorie wieder auf. Zusammen mit den Arbeiten von F. Neumann 1845/46 beherrschte das Webersche Gesetz die Elektrodynamik auf dem europäischen Festland bis in die 70er Jahre des vergangenen Jahrhunderts. ${ }^{11}$ Für Weber stellte die Konstante $c_{w}$ in seinem Gesetz eine wichtige Naturkonstante dar; für ihn war sie eine Art Grenzgeschwindigkeit. ${ }^{12}$ Nach dem Kohlrausch-Weber-Experiment ist ihre Grösse fast $\sqrt{2} \cdot$ Lichtgeschwindigkeit. Zur Überprüfung seines Gesetzes war Weber bereit, und er kam mit Kohlrausch überein, zunächst das Verhältnis von elektrostatisch und elektromagnetisch gemessener Ladung zu bestimmen. R. Kohlrausch war bei elektrostatischen Versuchen schon ein anerkannter Fachmann; 1853 hatte er das Sinuselektrometer konstruiert, mit dem auf einfache Weise das Verhältnis elektrostatischer Ladungen gefunden werden konnte. In diesem Gerät wird eine Magnetnadel und ihr Messingträger elektrisch aufgeladen. Die rücktreibende Kraft zur Messung der elektrostatischen Abstossung erzeugt das geomagnetische Feld. Weber hatte die elektromagnetischen Messmethoden und Messgeräte für damalige Verhältnisse zu hoher Vollkommenheit entwickelt. Es waren Nadelgalvanometer mit Ablenkspule, die ebenfalls die Horizontalkomponente des magnetischen Erdfeldes benutzten. Mit dem Geomagnetismus hatte sich Weber schon in den 30er Jahren, in seiner Periode der engen Zusammenarbeit mit Gauss, intensiv befasst.

Das Experiment verlief so: Eine Leidener Flasche wurde mit etwa dreissigtausend Volt (Elektrisiermaschine) aufgeladen, mit dem Knopf der Flasche eine mit Stanniol belegte grosse Kugel berührt, mit dem Sinuselektrometer das Verhältnis der in der Leidener Flasche zurückgebliebenen und der auf die Stanniolkugel geflossenen Ladung bestimmt, und die Leidener Flasche über das Nadelgalvanometer, das hier die Funktion eines Stossgalvano- 
meters hatte, entladen. Von der stanniolbelegten Kugel wurde der Standkugel der Coulombschen Drehwaage eine durch die Radien der Kugeln festgelegte Elektrizitätsmenge abgegeben und in der Drehwaage durch Torsion gemessen. Auf weitere Überlegungen, wie z. B. das Erfassen der Influenzwirkung oder das Dazwischenschalten einer Wassersäule, um die Entladungszeit zu dehnen und so Funkenüberschläge zu vermeiden, soll nicht weiter eingegangen werden. Mit dem Experiment sollte das Verhältnis zwischen dem «mechanischen Maass» der elektrischen Ladung oder auch der Stromstärke und dem elektromagnetischen Masse dieser Grössen gefunden werden. Es ergab sich $1,554 \cdot 10^{11} \mathrm{mms}^{-1}$; als Basiseinheiten benutzten Gauss und Weber immer mm, mg und s. Das «mechanischen Maass» ist aber doppelt so gross wie das eigentliche elektrostatische Mass. Weber hatte immer den elektrischen Doppelstrom vor Augen, und beim «mechanischen Maass» floss sowohl eine positive als auch eine negative elektrostatische Ladungseinheit durch den Leiterquerschnitt. ${ }^{13}$ Die Zahl muss deshalb mit zwei multipliziert werden, und man kommt so zu der von Fizeau gemessenen Lichtgeschwindigkeit. Merkwürdigerweise hat W. Weber diese Tatsache nie erwähnt. Er war ganz auf seine Konstante $c_{w}$ in seinem Gesetz fixiert. Zu dem von R. Kohlrausch und W. Weber gefundenen Wert schrieb Friedrich Kohlrausch (Sohn von R. Kohlrausch), der das Werk des ihm väterlich-freundschaftlich gesonnenen Weber fortsetzte: ${ }^{14}$

«So erscheint die Annäherung des ersten Resultates an den wahren, damals nicht einmal der Grössenordung nach bekannten Wert als eine bewundernswerte Leistung. Nur durch das Zusammenwirken der damaligen beiden Meister in den elektromagnetischen und den elektrostatischen Methoden ermöglicht, bildet sie einen klassischen Abschluss der Messungen mit Reibungselektrizität.»

Wie schon erwähnt, sah Maxwell in dem Ergebnis sofort die Lichtgeschwindigkeit. Es sei jedoch bemerkt, dass G. R. Kirchhoff vier Jahre davor die Fortpflanzungsgeschwindigkeit einer elektrischen Welle in einem Draht zu $\mathrm{c}_{\mathrm{w}} / \sqrt{2}$ (also etwa gleich der Lichtgeschwindigkeit) ebenfalls angegeben hatte. ${ }^{15}$ Auch B. Riemann (1826-1866) verfolgte Ende der 50er Jahre das Ziel, elektromagnetische Vorgänge mit Hilfe einer endlichen Ausbreitungsgeschwindigkeit, nämlich der des Lichts, zu beschreiben. ${ }^{16}$ Anscheinend war ihm der damals noch nicht veröffentlichte Brief von Gauss an Weber vom 19. März 1845 bekannt, in dem Gauss bedauerte, dass es ihm nicht gelingen wollte, die elektrodynamischen Kräfte aus einer ähnlich wie beim Licht sich fortpflanzenden Wirkung herzuleiten. ${ }^{17}$ Aus der Personalakte Riemanns geht hervor, dass W. Weber die Ernennung zum ausserordentlichen Professor 
Anfang März 1857 mit dem Hinweis empfahl, dass Riemann seit Jahren damit beschäftigt sei, Optik und Elektrizität miteinander zu verbinden, und dies wohl «Epoche» machen würde. ${ }^{18}$

Verwunderlich erscheint, dass Webers «Grenzgeschwindigkeit», bei der die elektrostatische Kraft gleich der elektrodynamischen ist, die Grösse $\sqrt{2} \cdot$ Lichtgeschwindigkeit hat. Weber geht dabei von zwei hypothetischen, in einer geraden Linie hintereinander liegenden Stromelementen aus bzw. von zwei Elektrizitätsteilchen, die sich in ihrer Verbindungsgeraden bewegen. Maxwell machte in seinem «Treatise» (1873) ein ähnliches Gedankenexperiment und definierte eine «kritische Geschwindigkeit», die gleich der Lichtgeschwindigkeit ist. Er lässt dabei zwei mit Elektrizität gleichen Vorzeichens belegte parallele Flächen sich in ihren Ebenen mit der Geschwindigkeit $\mathbf{v}$ verschieben. ${ }^{19}$ Es entstehen so zwei parallele Flächenströme, die sich magnetisch anziehen. Für das Verhältnis von elektrostatischer und elektromagnetischer Kraft findet er $\mathrm{c}_{\mathrm{vL}}^{2} / \mathrm{v}^{2}$, wobei $\mathrm{c}_{\mathrm{VL}}$ die Vakuumlichtgeschwindigkeit ist. Wird $\mathbf{v}$ gleich $\mathrm{c}_{\mathrm{vL}}$, sind die Kräfte gleich gross. Einen entsprechenden Gedankenversuch kann man mit zwei elektrischen Teilchen machen, sie müssen allerdings entgegengesetzt geladen sein und sich in entgegengesetzter Richtung parallel zueinander mit der relativen Geschwindigkeit v bewegen. Auch hier erhält man dann für das Verhältnis der Kräfte den Quotienten $c_{\mathrm{VL}}^{2} / \mathrm{v}^{2} .{ }^{20}$ In seinem «Treatise» gebrauchte Maxwell bei seiner Schreibweise des Weberschen Gesetzes als Konstante nicht $c_{w}$, sondern $c_{\mathrm{VL}}$. Vermutlich wollte er damit auch an die Verdienste Webers bei der Schaffung der absoluten Masssysteme erinnern. Mit der «kritischen Geschwindigkeit» deutet sich offenbar schon die besondere Rolle an, die der Quotient aus den Quadraten von v und der Lichtgeschwindigkeit in der späteren speziellen Relativitätstheorie spielen sollte. Leider war diese Doppelbedeutung der Bezeichnung «Weber-Konstante» Anlass zu mancher Verwirrung. ${ }^{21}$

W. Thomson und Maxwell befassten sich in den 60er Jahren eingehend mit den absoluten elektrischen Masssystemen, deren Grundideen auf Gauss zurückgehen und die Weber aufgenommen, weiter ausgearbeitet und experimentell umgesetzt hatte. Die aufkommende Elektrotechnik verlangte verbindliche Masse. Auf Thomsons Vorschlag hin bildete die British Association eine Kommission zur Festlegung praxisnaher Masseinheiten. Man entschied sich für Webers elektromagnetisches System und wählte geeignete Zehnerpotenzen aus. Auf dieses System geht letztlich dank der auf dem Ersten Elektrikerkongress in Paris (1881) gefassten Beschlüsse auch unser heutiges MKSA-System bzw. die SI-Einheiten zurück. ${ }^{22}$ 
Zuerst wurde von der British Association die Widerstandseinheit festgelegt. Auch hier griff man auf eine Webersche Methode der Widerstandsbestimmung zurück und verfeinerte sie. ${ }^{23}$ Maxwell schuf in jenen Jahren die Dimensionslehre in Anlehnung an Fourier und Gauss und er zeigte, welche Rolle die Lichtgeschwindigkeit für die gegenseitige Umrechnung der elektrischen Masse spielte. ${ }^{24}$ Noch wichtiger aber war für Maxwell die Bestimmung der Lichtgeschwindigkeit aus rein magnetischen und elektrischen Messungen; die Physiker sprachen hier von «v-Bestimmungen». ${ }^{25}$ Die Gültigkeit der Maxwellschen elektromagnetischen Lichttheorie hing ja damit zusammen. Maxwell gab dazu mehrere experimentelle Möglichkeiten an, und Ende der 60er Jahre führten er und W. Thomson solche «v-Bestimmungen» auch durch. Das Ergebnis bei Thomson war $\mathrm{v}=2,82 \cdot 10^{8} \mathrm{~ms}^{-1}$ und bei Maxwell $2,88 \cdot 10^{8} \mathrm{~ms}^{-1}$. W. Weber und R. Kohlrausch hatten (1855) $3,108 \cdot 10^{8} \mathrm{~ms}^{-1}$ erhalten. Durch direkte terrestrische Bestimmung der Lichtgeschwindigkeit hatte, wie schon erwähnt, Fizeau (1849) $3,15 \cdot 10^{8} \mathrm{~ms}^{-1}$ gefunden und danach Foucault (1850) 2,98 $10^{8} \mathrm{~ms}^{-1}$. Eine Konvergenz von $\mathrm{v}$ mit $\mathrm{c}_{\mathrm{vL}}$ schien damit gesichert. Erstaunlich bleibt, dass bei Thomsons und Maxwells Versuchen trotz aller Raffinesse keine grössere Genauigkeit erzielt wurde als 13 Jahre zuvor bei Kohlrausch und Weber. Durch sorgfältige Untersuchungen von Physikern verschiedener Nationen wurde in jahrelanger Arbeit die Konvergenz von $\mathrm{v}$ und $\mathrm{c}_{\mathrm{VL}}$ dann endgültig gesichert.

Zum Schluss soll noch einmal auf den für die Entwicklung der Maxwellschen Elektrodynamik und Lichttheorie so wichtigen magneto-optischen Effekt von Faraday eingegangen werden. Auch auf dem Festland hatte 1858 Carl Neumann (1832-1925) den Effekt in Anlehnung an das Webersche Gesetz mit Hilfe eines Wirkungsgesetzes zwischen Elektrizitäts- und Ätherteilchen theoretisch zu deuten versucht. ${ }^{26}$ Sein Vater Franz Neumann (1798-1895), dem nicht nur die Elektrizitätslehre, sondern auch die Optik, insbesondere die Kristallphysik, bahnbrechende Arbeiten verdankt, hatte dazu den Anstoss gegeben. Während bei Maxwell die Drehung der Polarisationsebene einzig und allein durch Vorgänge im Äther bewirkt wird, bringt C. Neumann die Materie mit ihren Molekularströmen, nach W. Weber um einen Kern kreisende elektrische Teilchen, mit ins Spiel. ${ }^{27}$ H. A. Lorentz, der die Maxwellsche Elektrodynamik um 1892 durch Hinzunahme der älteren Vorstellungen Webers ergänzte, um damit den individuellen Eigenschaften der Materie, wie z.B. der Dispersion und den Spektren gerecht zu werden, drückte sein Erstaunen darüber aus, wie nahe C. Neumanns Deutung der seinigen kommt. ${ }^{28}$ Konnten bei C. Neumann die Molekularströme (Doppel- 
ströme positiver und negativer elektrischer Teilchen) keine Lichtwellen erregen, sprach dagegen schon 1862 W. Weber den Gedanken aus, dass dies sehr wohl der Fall sein könnte, wenn man nur eine Elektrizitätsart sich periodisch bewegen liesse und die andere Elektrizitätsart mit dem Kern des Moleküls verschmelze. Weber hatte damit die Ampèreschen Molekularströme in ein Planetensystem aufgelöst. ${ }^{29}$ J. K. F. Zöllner, ein eifriger Verfechter der Weberschen Theorie, vermutete in seinen «Principien einer elektrodynamischen Theorie der Materie» (1876), dass damit «Zahl und Lage der Spektrallinien der chemischen Elemente» erklärt werden könnte. ${ }^{30}$

Die obigen Ausführungen versuchten darzulegen, dass das KohlrauschWeber-Experiment von 1855 einer der entscheidenden Anstösse für Maxwell gewesen war, seine elektromagnetische Lichttheorie zu entwickeln. Daneben wollten wir zeigen, dass in der auf Maxwells Werk folgenden Elektronen- und Atomtheorie Ideen der Weberschen Elektrodynamik wieder aufgenommen wurden.

\section{Literatur und Anmerkungen}

1 Die Abhandlung stellt eine Erweiterung des Vortrages dar, gehalten bei der Eröffnung der Sonderausstellung «Die Gebrüder Weber - Wegbereiter interdisziplinärer Forschung» der Wilhelm-Weber-Gesellschaft e.V. und der Martin-Luther-Universität, Halle-Wittenberg, am 23. September 1993 in Halle.

2 Scientific Papers of J. Cl. Maxwell, ed. by W.D. Niven 2 Vols. 1890, hier Vol.I S.451. Deutsch in Ostwald's Klassiker der exakten Wissenschaften Nr.102. Über Physikalische Kraftlinien. Hrsg. von L. Boltzmann. Leipzig 1898.

3 R. Kohlrausch und W. Weber: Über die Elektrizitätsmenge, welche bei galvanischen Strömen durch den Querschnitt der Kette fliesst. Annalen für Physik und Chemie, hrsg. von J. C. Poggendorff. Bd. 99 (1856) S. 10; auch in Wilhelm Webers Werke. 6 Bde. Hrsg. von der Kgl. Ges. d. Wiss. zu Göttingen. Berlin 1892-1894, hier Bd. 3 S. 597 - Die umfangreichere Abhandlung der beiden Autoren ist: Elektrodynamische Maassbestimmungen, insbesondere Zurückführung der Stromintensitätsmessungen auf mechanisches Maas: (1857). Weber Werke Bd.3 S.609. - Das Vorwort bei der Übergabe der Abhandlung (1855) in Weber Werke Bd. 3 S. 591.

4 H. Fizeau, Compt. rend. T. 29 (1849) S. 90 und Ann. d. Phys. u. Ch. hrsg. von J.C. Poggendorff, Bd. 79 (1850), S. 164.

5 L. Campbell und W. Garnett: The Life of James Clerk Maxwell, with selections from his correspondence and occasional writings. New ed. London 1884, S. 243 und 244. Siehe dazu auch den Brief Maxwells vom 10. Dez. 1861 an W. Thomson. In: J. Larmor: The origins of Clerk Maxwell's electric ideas as described in familiar letters to W. Thomson. Proceedings of the Cambridge Philosophical Society Vol. 32 (1936) S. 728.

6 R.P. Feynman: Lectures on Physics. Mainly Electromagnetism and Matter. 4. Aufl. Mass. 1966. - I. Tolstoy: J. Cl. Maxwell. A Biography. Edinburgh 1981. 
7 Siehe dazu K. H. Wiederkehr: Wilhelm Weber und die Entwicklung in der Geomagnetik und Elektrodynamik. Mitteil. der Gauss-Gesellschaft Nr.29, S.63. Göttingen 1992.

8 Ostwalds Klass. Nr. 102, S.67.

9 Scientific Papers of J. Cl. Maxwell I, S. 526.

10 W. Weber: Elektrodynamische Maassbestimmungen, insbesondere über ein allgemeines Grundgesetz der elektrischen Wirkung (1846). Weber Werke Bd. 3 S. 25-214, s. S. $138 \mathrm{ff}$.

11 F.Neumann: Die mathematischen Gesetze der inducirten elektrischen Ströme (1845). Ostwalds Klass. Nr. 10. Hrsg. von C. Neumann. Leipzig 1889. - Ders.: Über ein allgemeines Princip der mathematischen Theorie inducirter elektrischer Ströme (1847). Ostw. Kl. Nr. 36. Hrsg. von C. Neumann. Leipzig 1892.

12 Siehe dazu W. Weber Werke Bd.3, S.594 und 651. - Auch beim Gravitationsgesetz sollte eine Korrektur durch den Faktor $\mathrm{v}^{2} / \mathrm{c}_{\mathrm{w}}^{2}$ erfolgen. Siehe dazu F. Rosenberger: Geschichte der Physik, T. 3, Braunschweig 1887-1890, S.583.

13 Pogg. Ann., Bd. 99 (1856), S. 11 und Weber Werke Bd. 3 S. 597.

14 R. Kohlrausch und W. Weber: Fünf Abhandlungen über absol. elektr. Strom- und Widerstandsmessung. Hrsg. von F. Kohlrausch. Ostwalds Klass. Nr.142. Leipzig 1904. Zitat S. 115. Vgl. auch Ostw. Klass., Neue Folge Nr. 3, Über die Einführung absoluter elektrischer Masse. Kommentiert von K. H. Wiederkehr, Braunschweig 1968, S. 114.

15 G.R. Kirchhoff: Über die Bewegung der Elektrizität in Drähten. Annalen der Physik. Hrsg. von J. C. Poggendorff. Bd. 100 (1857), S. 193. In seiner Abhandlung: Elektrodynamische Maassbestimmungen, insbesondere über elektrische Schwingungen, Werke Bd.4, S. 105, hierzu S. 157, kommentierte Weber das Ergebnis von Kirchhoff und ging dann auf die Vermutung eines eventuellen Zusammenhanges zwischen elektrischen Wellen und Lichtwellen ein.

16 B. Riemann: Ein Beitrag zur Elektrodynamik (1858). Gesammelte mathematische Werke und wissenschaftlicher Nachlass. Hrsg. von H. Weber, Leipzig 1876, S.270. Riemann hatte die Abhandlung zurückgezogen; sie wurde aber später in den Ann. d. Phys. 1867, Bd. 131 veröffentlicht.

17 C.F. Gauss: Werke V. Hrsg. von der Kgl. Ges. d. Wiss. zu Gött. 1867, S. 627.

18 K.H. Wiederkehr: Freunde und Förderer Bernhard Riemanns. Zwei Briefe aus seinen letzten Lebensjahren. Mitteil. Nr. 27 (1990) der Gauss-Ges. Gött., S. 75.

19 J. Cl. Maxwell: Treatise on Electricity and Magnetism.2 Vols. London 1873. Deutsch von B. Weinstein, Lehrb. d. Elektr. u. d. Magn., 2 Bde. Berlin 1883. Hierzu Bd. 2 S. 518.

20 Siehe dazu K. H. Wiederkehr: Wilhelm Webers Stellung in der Entwicklung der Elektrizitätslehre. Diss. Hamburg 1960, S. 130.

21 Lehrb. d. Elektr. u. d. Magn., Bd.2, Art. 853. So bringt anscheinend auch C. W. F. Everitt in seiner Kurzbiographie zu Maxwell in Dictionary of Scientific Biography Bd. IX (1974) auf S. 209 einiges durcheinander. - Und bei O. Blumtritt : Zur Genese der elektromagnetischen Feld theorie, Hildesheim 1986, werden beide Bedeutungen der «Weber-Konstanten» nebeneinander benutzt. Das letzte Glied in der Klammer für die Formel dF auf S. 34 müsste $+\frac{\mathrm{r}}{\mathrm{c}_{\mathrm{vL}}^{2}} \cdot \frac{\mathrm{d}^{2} \mathrm{r}}{\mathrm{dt}^{2}}$ heissen. Daneben stellt Blumtritt die Behauptung auf, dass W. Weber auf der Existenz eines magnetischen Fluidums beharrte und Elektrizität und Magnetismus streng von einander trennen wollte. Das Gegenteil ist richtig. 
22 Bericht über den Kongressverlauf, Elektrotechnische Zeitschr. Jg. 2 (1881) und Annalen der Physik hrsg. v. G. Wiedemann Bd.14 (1881), S. 708 und Anhang B. - Siehe auch U. Stille: Messen und Rechnen in der Physik. Braunschweig 1955, S. 213 und S.German und P. Drath: Handbuch der SI-Einheiten, Braunschweig 1979, S. 141.

23 Report 33rd Meet. British Association for the Advancement of Science. Newcastle 1863, London 1864, S.111. Auch in Maxwell, Lehrb. d. Elektr. u. d. Magn., Bd.2, S.510.

24 J. Cl. Maxwell and F. Jenkin: On the Elementary Relations between Electrical Measurements. In: Philosophical Magazine 4. Ser. Vol. 29 (1865) S. 436 und 507.

25 Maxwell, Scient. Papers. Vol.2, S.125, ebenso Lehrb. d. Elektr. etc., Bd.2. S.522, - und G. Wiedemann: Die Lehre von der Elektricität. 4 Bde. 2. Aufl. Braunschweig 1893-1898, hier Bd.4, S. 755.

26 C. Neumann: Explicare tentatur quomodo fiat ut lucis planum polarisationis per vires electricas vel magneticas declinetur. Hales Saxonum 1858. Deutsch: Die magnetische Drehung der Polarisationsebene des Lichtes. Halle 1863

27 Maxwell, Lehrb. d. Elektr. etc., Bd.2, S.570, und G. Wiedemann, Die Lehre v.d. Elektr., Bd.3, S.1111.

28 H. A. Lorentz: Theorie der magneto-optischen Phänomene (1909). In: Encyklopädie der Mathematischen Wissenschaften. 5.Bd. (Physik) 3. Teil, Leipzig 1909-1926, S. 256.

29 W. Weber: Zur Galvanometrie (1862). Werke Bd.4, S.17.

30 J.C. Fr. Zöllner: Principien einer elektrodynamischen Theorie der Materie. Leipzig 1876. Vorrede S.XXI.

Dr. rer.nat. habil. Karl Heinrich Wiederkehr

Studiendirektor i. R. und Privatdozent

für Geschichte der Naturwissenschaften

Birkenau 24

D-22087 Hamburg 